\title{
Controle de Qualidade dos monitores de interpretação de imagens radiológicas digitais: Uma revisão
} Mariana S. Favero ${ }^{1}$, Adriano S. Goulart ${ }^{1}$

${ }^{1}$ PhyMED - Consultores em Física Médica e Radioproteção LTDA, Porto Alegre, Brasil

\begin{abstract}
Resumo
O desempenho de monitores tem grande importância na qualidade da imagem de sistemas radiográficos digitais. Em ambientes sem filmes, tornou-se necessário implementar testes de aceitação e controle de qualidade em monitores usados para interpretação de imagens médicas. Os monitores dedicados ao radiodiagnóstico devem fornecer informações que representam pequenas diferenças em atenuação de raios $X$ ou pequenas diferenças de alguma região anatômica de interesse. Isso deve resultar também em pequenas diferenças em luminância de uma imagem representada. Os fatores que afetam a qualidade das imagens médicas são contraste, ruído, resolução, artefatos e distorções. Por isso, um monitor deve possuir características específicas, para que tornem possível ao médico radiologista realizar uma avaliação que leve ao melhor diagnóstico. Com base na necessidade de avaliar monitores para diagnóstico nas mais diversas aplicações radiológicas, esse trabalho visa apresentar um resumo para a execução e padronização dos testes que são recomendados pela publicação AAPM Report 03.
\end{abstract}

Palavras-chave: radiologia diagnóstica, controle de qualidade, monitores de intrepretação.

\begin{abstract}
The performance monitors has great importance in image quality of digital radiographic systems. In environments without films, it became necessary to implement acceptance testing and quality control monitors used for interpretation of medical images. The monitors dedicated to radiodiagnostic should provide information that represent slight differences in $x$-ray attenuation or minor differences in some anatomical region of interest. This should also result in small differences in luminance of an image represented. Factors affecting the quality of medical imaging are contrast, noise, resolution, artifacts and distortions. Therefore, a monitor must have specific characteristics, making it possible for the observer to carry out an assessment that leads to better diagnosis. Based on the need to evaluate diagnostic monitors in various radiological applications, this paper presents a summary for implementation and standardization of tests that are recommended by the publication AAPM Report 03.
\end{abstract}

Keywords: diagnostic radiology, quality control, display monitors.

\section{Introdução}

A utilização de monitores para visualização de imagens médicas teve início nos anos 70. Primariamente, os monitores estavam associados à imagem de tomografia computadorizada, e posteriormente, também a ecografia, radiologia digital e ressonância magnética. Os monitores de diagnóstico por imagem são o último elo na cadeia da imaginologia médica. Com a variabilidade de tecnologias e degradação física com o tempo de vida, estes monitores devem ser cuidadosamente avaliados de modo a demonstrar uma performance correta e estável ao longo do tempo. Os monitores usados para visualização e diagnóstico de imagens médicas devem por isso ser regularmente verificados para que se mantenha um sistema com a qualidade que é exigida, dada a função que desempenham [1]. Monitores de classe primária são utilizados para a interpretação de imagens médicas e são normalmente utilizados em determinadas especialidades médicas. Monitores de classe secundária são utilizados para a visualização de imagens médicas que não tenham como fim a interpretação médica. Nessa classe de monitores estão também as estações de trabalho de sistemas digitais onde as imagens são ajustadas antes de serem enviadas para o PACS ou impressora. Esses monitores dedicados ao radiodiagnóstico devem fornecer informações que representam pequenas diferenças em atenuação de raios $X$ ou pequenas diferenças de alguma região anatômica de interesse. Isso deve resultar também em pequenas diferenças em luminância de uma imagem representada. Por isso, um monitor deve possuir características específicas, tais como resolução espacial e de contraste, ruído, luminância, distorção geométrica e reflexão apropriada que tornem possível ao observador realizar uma avaliação que leve ao melhor diagnóstico [2]. Os principais testes para a avaliação do desempenho de monitores são: distorção geométrica da imagem no monitor, reflexão na tela do monitor, uniformidade da luminância, resposta da luminância e de contraste, resolução do monitor, ruído na imagem e velamento por reflexão interna da tela do monitor [3]. O objetivo desse trabalho é apresentar um resumo para a execução e padronização dos testes que são recomendados pela publicação Report 03 da Associação Americana de Físicos em Medicina, do inglês American Association of Physics in Medicine (AAPM). 


\section{Materiais e Métodos}

Com base na necessidade de avaliar monitores para diagnóstico nas mais diversas aplicações radiológicas, foi realizada uma pesquisa bibliográfica e realizado um guia para auxiliar na execução e padronização dos testes que são recomendados pela publicação Report 03. Nesse trabalho será apresentado um resumo, com os métodos de análise, que podem ser do tipo qualitativo ou quantitativo bem como a periodicidade, as ferramentas necessárias para execução, os níveis de referência e a sugestão de ação para cada teste recomendado. Sendo aplicável para os monitores de raios catódicos (CRT) e monitores de cristal líquido (LCD). Os monitores de visualização utilizados na área médica, segundo o Colégio Americano de Radiologia (ACR), podem ser classificados como primário ou secundário. Os monitores classificados como primários são aqueles utilizados para interpretação das imagens médicas, e tipicamente utilizados em radiologia e em certas especialidades médicas. Os secundários são utilizados para visualização das imagens médicas, normalmente utilizados pela equipe médica e especialistas que não são radiologistas, e utilizados após o fornecimento de um relatório interpretativo da análise das imagens. Na classe dos monitores secundários, existem os monitores dos consoles dos operadores, que permitem ajustes nas imagens antes delas serem enviadas para o sistema de Arquivamento e Comunicação de Imagens, do inglês Picture Archiving and Communication System (PACS) [4].

\section{Resultados}

Os métodos de avaliação para os sistemas de visualização de imagens médicas podem ser:

- Avaliação Qualitativa: onde são realizadas análises visuais através dos padrões da imagem;

- Avaliação Quantitativa: onde são realizadas análises com a utilização de instrumentos de medidas;

A tabela 1 apresenta os testes qualitativos, 0 padrão de imagem necessário e a descrição do teste realizado.

A tabela 2 apresenta um resumo do teste de controle de qualidade diário, que pode ser executado pelo usuário operador do monitor.

A tabela 3 apresenta um resumo dos testes de controle de qualidade mensal/trimestral, que pode ser executado pelo físico médico, ou pelo técnico com supervisão do físico médico.

A tabela 4 apresenta um resumo dos testes de controle de qualidade anual, que pode ser executado pelo físico médico, ou pelo técnico com supervisão do físico médico.

\section{Discussão}

O desempenho de monitores tem grande importância na qualidade da imagem de sistemas radiográficos digitais. A fim de se garantir o melhor diagnóstico é fundamental que seja implementado nos serviços de diagnóstico por imagem, um programa contemplando também os monitores de visualização.

\section{Conclusões}

Esse trabalho apresenta um resumo bibliográfico para execução e padronização dos testes que são recomendados pela publicação Report 03 da Associação Americana de Físicos em Medicina, do inglês American Association of Physics in Medicine (AAPM), a metodologia para execução de cada teste está sendo aplicada em diferentes serviços, onde estão sendo implementados os testes de controle de qualidade na rotina. Estudos recentes apontam a importância em realizar 0 acompanhamento dos monitores utilizados ao longo dos anos. A perspectiva para próximos trabalhos é apresentar os resultados dessas avaliações na qualidade para diferentes serviços ao longo dos anos.

\section{Referências}

1. Pinto M, Pedro M, Santos A, Saraiva A. Controle de qualidade em monitores de diagnóstico por imagem e iluminância nos espaços de pós-processamento em serviços de imaginologia. Radiol Bras. 2012; 45(1):29-34.

2. Ribeiro DR, Furquim TC. Estudo do desempenho de monitores LCD utilizados em radiologia digital. Rev Imagem (Online) 2011;33(1/2):1-6.

3. Furquim TAC, Costa PR. Garantia de qualidade em radiologia diagnóstica. Revista Brasileira de Física Médica. 2009; 3(1): 91-99.

4. Medeiros GS. Desenvolvimento de um protocolo de controle da qualidade de monitores para visualização de imagens radiológicas digitais. [Trabalho de Conclusão de Curso]. Porto Alegre: Faculdade de Física Pontifícia Universidade Católica do Rio Grande do Sul; 2007.

\section{Contato:}

Mariana Saibt Favero

Avenida Osvaldo Aranha, 1180 salas 303/304

(PhyMED Consultores em Física Médica e

Radioproteção)

E-mail:mariana@phymed.com.br 
Tabela 1 - Métodos de Avaliação

\begin{tabular}{|c|c|c|}
\hline Método & Padrão de Imagem & Descrição \\
\hline & TG18-QC & Resolução, distorção e artefatos \\
\hline & TG18-BR & "Padrão de Briggs" - Contraste e resolução \\
\hline & TG18-PQC & Resolução e Contraste \\
\hline & TG18-CT & Resposta de Luminância \\
\hline & TG18-UN & Uniformidade \\
\hline & TG18-AD & Reflexão Difusa \\
\hline & TG18-MP & Resolução bit-profundidade \\
\hline \multirow[t]{8}{*}{ Qualitativo } & TG18-CX & "Padrão Cx" - Uniformidade e Resolução \\
\hline & TG18-LPH & Barras Horizontais em três níveis de luminância \\
\hline & TG18-LPV & Barras Verticais em três níveis de luminância \\
\hline & TG18-AFC & "Padrão AFC" - Contraste \\
\hline & TG18-GV & "Padrão Dark-spot" - Baixo Contraste \\
\hline & TG18-CH & Teste anatômico - Radiografia de Tórax \\
\hline & TG18-KN & Teste anatômico - Radiografia de Joelho \\
\hline & TG18-MN & Teste anatômico - Mamografia \\
\hline \multirow{11}{*}{ Quantitativo } & TG18-QC & Distorções Geométricas \\
\hline & TG18-PQC & $\begin{array}{l}\text { Padrões horizontais e verticais de alto e baixo } \\
\text { contraste }\end{array}$ \\
\hline & TG18-LN & Curva Característica do Monitor \\
\hline & TG18-UNL & Uniformidade da Luminância \\
\hline & TG18-RH & Avaliação quantitativa da resolução \\
\hline & TG18-RV & Avaliação quantitativa da resolução \\
\hline & \multirow{3}{*}{ TG18-PX } & Caracterização quantitativa da resolução \\
\hline & & Caracterização do perfil de luminância do pixel \\
\hline & & Avaliação do ruído em cinco regiões uniformes \\
\hline & TG18-GQ & "Padrão Dark-spot" - Medição do ruído \\
\hline & TG18-GA & Tamanho variável do "Padrão Dark-spot" \\
\hline
\end{tabular}


Tabela 2 - Teste de Controle de Qualidade diário para monitores de visualização de imagens médicas

\begin{tabular}{|c|c|c|c|c|}
\hline \multirow{2}{*}{ Teste } & \multicolumn{2}{|c|}{ Ferramentas Necessárias } & \multirow{2}{*}{$\begin{array}{l}\text { Níveis de } \\
\text { Referência }\end{array}$} & \multirow{2}{*}{ Sugestão de Ação } \\
\hline & Equipamento & Padrão de imagem & & \\
\hline Avaliação Visual Geral & Não necessário & $\begin{array}{l}\text { TG18-QC ou } \\
\text { imagens anatômicas }\end{array}$ & Visual & $\begin{array}{l}\text { Realizar uma avaliação } \\
\text { mais detalhada. }\end{array}$ \\
\hline
\end{tabular}

Tabela 3 - Testes de Controle de Qualidade mensal/trimestral para monitores de interpretação de imagens médicas

\begin{tabular}{|c|c|c|c|c|}
\hline \multirow[b]{2}{*}{ Teste } & \multicolumn{2}{|c|}{ Ferramentas Necessárias } & \multirow[b]{2}{*}{ Níveis de Referência } & \multirow[b]{2}{*}{ Sugestão de Ação } \\
\hline & Equipamentc & $\begin{array}{l}\text { Padrão de } \\
\text { imagem }\end{array}$ & & \\
\hline Distorção Geométrica & $\begin{array}{l}\text { Não } \\
\text { necessário }\end{array}$ & TG18-QC & Visual & $\begin{array}{l}\text { Realizar uma avaliação } \\
\text { mais detalhada. }\end{array}$ \\
\hline Reflexão & Fotômetro & TG18-AD & $\begin{array}{c}\text { Lmín } \geq 1,5 \text { Lamb } \\
\text { Valor Ideal: Lmín } \geq 4 \text { Lamb }\end{array}$ & $\begin{array}{l}\text { Reajuste do nível de } \\
\text { luminância ambiental. }\end{array}$ \\
\hline \multirow{3}{*}{ Resposta de Luminância } & \multirow{3}{*}{ Fotômetro } & TG18-LN & $\begin{array}{c}\text { Para monitores primários: } \\
\text { Lmáx } \geq 170 \mathrm{~cd} / \mathrm{m}^{2} \\
\text { Para monitores secundários: } \\
\text { Lmáx } \geq 100 \mathrm{~cd} / \mathrm{m}^{2}\end{array}$ & \multirow{3}{*}{$\begin{array}{l}\text { Reajuste, reparo } \\
\text { recalibração ou } \\
\text { substituição para } \\
\text { repetição dos testes. }\end{array}$} \\
\hline & & TG18-CT & $\begin{array}{c}\text { Para monitores primários: } \\
\text { RC } \geq 250 \\
\text { Para monitores secundários: } \\
R C \geq 100\end{array}$ & \\
\hline & & TG18-MP & $\Delta$ Lmáx $\leq 10 \%$ & \\
\hline \multirow{2}{*}{ Dependência de Luminância } & \multirow{2}{*}{ Fotômetro } & TG18-UN & \multirow{2}{*}{ Uniformidade $\leq 30 \%$} & \multirow{2}{*}{$\begin{array}{l}\text { Reajuste, reparo ou } \\
\text { substituição para } \\
\text { repetição dos testes. }\end{array}$} \\
\hline & & TG18-UNL & & \\
\hline Resolução & Lupa & TG18-CX & $\begin{array}{c}\text { Para monitores primários: } \\
0 \leq \mathrm{Cx} \leq 6 \\
\text { Para monitores secundários: } \\
0 \leq \mathrm{Cx} \leq 4\end{array}$ & $\begin{array}{l}\text { Ajuste de foco, reparo } \\
\text { ou substituição para } \\
\text { repetição dos testes }\end{array}$ \\
\hline
\end{tabular}

Tabela 4 - Testes de Controle de Qualidade anual para monitores de visualização de imagens médicas

\begin{tabular}{|c|c|c|c|c|}
\hline \multirow[b]{2}{*}{ Teste } & \multicolumn{2}{|c|}{ Ferramentas Necessárias } & \multirow[b]{2}{*}{ Níveis de Referência } & \multirow[b]{2}{*}{ Sugestão de Ação } \\
\hline & Equipamento & $\begin{array}{l}\text { Padrão de } \\
\text { imagem }\end{array}$ & & \\
\hline Distorção Geométrica & $\begin{array}{l}\text { Régua de } \\
\text { Precisão }\end{array}$ & TG18-QC & $\begin{array}{c}\text { Para monitores primários: } \\
\text { Desvio } \leq 2 \% \\
\text { Para monitores secundários: } \\
\text { Desvio } \leq 5 \%\end{array}$ & $\begin{array}{l}\text { Reajuste, reparo ou } \\
\text { substituição para } \\
\text { repetição dos testes. }\end{array}$ \\
\hline Ruído & $\begin{array}{l}\text { Não } \\
\text { necessário }\end{array}$ & TG18-AFC & $\begin{array}{l}\text { Para monitores primários: } \\
\text { É admissível não serem } \\
\text { visualizados pontos no } 1^{\circ} \\
\text { quadrante. } \\
\text { Para monitores secundários: } \\
\text { É admissível não serem } \\
\text { visualizados pontos nos } 1^{\circ} \text { e } 2^{\circ} \\
\text { quadrantes. }\end{array}$ & $\begin{array}{l}\text { Reajuste, reparo ou } \\
\text { substituição para } \\
\text { repetição dos testes. }\end{array}$ \\
\hline \multirow{2}{*}{ Saturação de Brilho } & \multirow{2}{*}{$\begin{array}{l}\text { Não } \\
\text { necessário }\end{array}$} & $\begin{array}{l}\text { TG18-GV e } \\
\text { TG18-GVN }\end{array}$ & $\begin{array}{l}\text { Para monitores primários: } \\
\text { Pelo menos, três estruturas. } \\
\text { Para monitores secundários: } \\
\text { Pelo menos, uma estrutura. }\end{array}$ & \multirow{2}{*}{$\begin{array}{l}\text { Reverificação da } \\
\text { resposta de luminância, } \\
\text { caso contrário } \\
\text { substituição. }\end{array}$} \\
\hline & & TG18-GQ & $\begin{array}{c}\text { Para monitores primários: } \\
\text { GR } \geq 400 \\
\text { Para monitores secundários: } \\
\text { GR } \geq 150 \\
\end{array}$ & \\
\hline Cromaticidade & $\begin{array}{l}\text { Não } \\
\text { necessário }\end{array}$ & TG18-UNL80 & Visual & Substituição. \\
\hline
\end{tabular}

